\title{
Networks of common property resources
}

\author{
Rahmi İlkılıç
}

Received: 31 October 2008 / Accepted: 2 February 2010 / Published online: 9 March 2010

(C) The Author(s) 2010. This article is published with open access at Springerlink.com

\begin{abstract}
A tragedy of commons appears when the users of a common resource have incentives to exploit it more than the socially efficient level. We analyze the situation when the tragedy of commons is embedded in a network of users and sources. Users play a game of extractions, where they decide how much resource to draw from each source they are connected to. We show that the network structure matters. The exploitation at each source depends on the centrality of the links connecting the source to the users. The equilibrium is unique and we provide a formula which expresses the quantities at an equilibrium as a function of a network centrality measure. Next we characterize the efficient levels of extractions by users and outflows from sources. We provide a graph decomposition which divides the network into regions according to the availability of sources. Then the efficiency problem can be solved region by region.
\end{abstract}

Keywords Commons · Networks · Nash equilibrium · Efficiency ·

Centrality measures

JEL Classification $\quad$ C62 $\cdot$ C72 $\cdot \mathrm{D} 85 \cdot \mathrm{Q} 20$

\footnotetext{
I am grateful to Antoni Calvó-Armengol for his guidance and supervision. I would like to thank to an anonymous referee whose comments helped improve the paper substantially. I also thank Francis Bloch, Olivier Bochet, Aytek Erdil, Ayça Giritligil, Guillaume Haeringer, Sjaak Hurkens, Matthew Jackson, Bettina Klaus and Jordi Massó. I gratefully acknowledge the support of the European Community via Marie Curie Grant PIEF-GA-2008-220181.
}

R. İlkılıç (ه)

Department of Economics, Maastricht University, 6200 MD Maastricht, The Netherlands e-mail: r.ilkilic@maastrichtuniversity.com 


\section{Introduction}

Since the analysis of a common property fishery by Gordon (1954) "the tragedy of the commons" has been studied extensively. ${ }^{1}$ Though one aspect that eluded the attention of the literature is the multiplicity of commons.

In a standard model of commons, there exists a single source exploited by many users. In reality, the most representative commons (e.g. pastures, forests, fisheries and sources of fresh water) are local, but numerous. The multiplicity of sources brings new political and economic questions.

In Spain, the drought years of 2006 and 2007 resulted in a shortage of fresh water. The efforts by the government to supply the south from the sources in the north started a legal and political debate. Both the autonomous regions and the central government claimed sovereignty over the sources. ${ }^{2}$ The proposed solutions involve the construction of new pipelines to transfer water and desalification plants to process sea water for agricultural use. The rainy year of 2008 abated the problem, but water supply and demand stay in a delicate balance at the mercy of the weather. ${ }^{3}$

The European Union implements a Common Fisheries Policy (CFP) to ensure the sustainable exploitation of the region's living marine resources. The European Commission implements conservation measures including quotas on member countries and fishing moratoriums on particular basins. In an effort to expand the available fishing basins, EU negotiates with third countries and buys access rights to fish in their sovereign waters. ${ }^{4}$

Although a great deal is known about a single common, there exists no theoretic model of multiple sources as exemplified above. In this paper, we will answer the two basic questions surrounding a tragedy of commons. We will determine the equilibrium when the users exploit the sources freely. We will then characterize the efficient allocation of resources.

We model a bipartite network, where links connect cities with sources. We look at the extraction game, where agents decide how much water to draw from each source they are connected to. The cities receive a value from consumption of the resource, but the extraction is costly. We assume that the value of consumption is concave and

\footnotetext{
1 Levhari and Mirman (1980), Benhabib and Radner (1992), Dutta and Sundaram (1993), Dockner and Sorger (1996) analyze the dynamic extraction of commons. Kremer and Morcom (2000), Gaudet (2002) study the storable commons which need not be consumed upon extraction. Rowat and Dutta (2007) analyze a common where the users have access to capital markets. On the empirical side, many real life examples have been discussed in Ostrom $(1991,1994)$ and Ostrom et al. (2002). They provide both theoretical and empirical analysis concerning possible solutions for the tragedy of the commons.

2 In addition, the better supplied north claimed the transfers would be harmful for the environment, jeopardizing the quality and the sustainability of their sources, while the south pressed their urgent need for water.

3 See Valdecantos (2005), Nash (2008) for more information on the problem and the debate.

4 See European Council (2002) for the basic regulation governing the CFP. The European Commission provides detailed information on the implementation of the policy in its fisheries web site (http://ec.europa. eu/fisheries).
} 
Fig. 1 The complete bipartite network of two cities and two sources

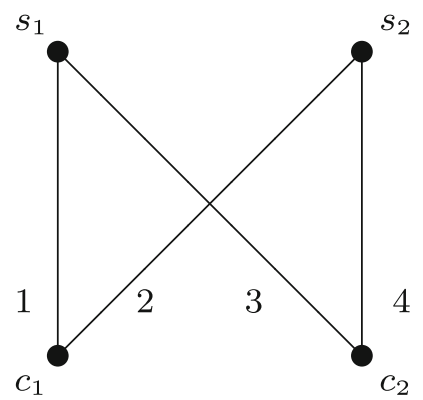

$g_{1}$

the cost of extraction at each source is a convex function of the total extraction at that source. ${ }^{5}$

We show the extraction of a city at a source does not only depend on the number of users she shares it with. It also depends on the number of sources their neighbors are linked to. And also on the number of users at the sources which their neighbors are linked to. The externalities diffuse through the paths ad infinitum. We write the equilibrium conditions as a linear complementarity problem and show uniqueness in Theorem 1. We interpret the equilibrium flows using the Katz-Bonacich centralities (Katz 1953; Bonacich 1987) of the links in Theorem 3.

The complementarities in the network are between the links. The flows on two different links are either strategic substitutes or complements. The sign of the complementarity is determined by the number of nodes between them.

For example in graph $g_{1}$, where $c_{1}, c_{2}$ are the cities and $s_{1}, s_{2}$ are the sources, the flows on links 1 and 2 are strategic substitutes (Fig. 1). They both supply to $c_{1}$. The inflow from one of them decreases the marginal value of water to $c_{1}$. This in turn decreases the incentive to extract via the other link. The flows on links 1 and 3 are also strategic substitutes. They connect $s_{1}$ to $c_{1}$ and $c_{2}$. The outflow from one of the links increases the marginal cost of extraction from $s_{1}$. This in turn decreases the incentive to extract via the other link. In brief, the flows on neighboring links are substitutes.

The flows on links 1 and 2 are strategic substitutes, idem the flows on links 2 and 4. This makes the flows on links 1 and 4 complements. The extraction from one increases the incentive to extract more from the other. The type of the complementarity between the flows on any two links can be determined in this manner by following a path connecting them. In general, the flows on links with an odd number of nodes between them are strategic substitutes and the flows on links with an even number of nodes

\footnotetext{
5 The assumptions of concave value of consumption (Smith 1968; Levhari and Mirman 1980; Dutta and Sundaram 1993; Dockner and Sorger 1996; Rowat and Dutta 2007) and convex cost of extraction (Gordon 1954; Smith 1968; Rowat and Dutta 2007) are familiar in the analysis of common property resources. Moreover, in dynamic models where the resource propagates with time (Benhabib and Radner 1992; Dutta and Sundaram 1993; Dockner and Sorger 1996), extraction diminishes both the current and all the future stocks. When discounted, this leads to a convex cost.
} 
between them are strategic complements. ${ }^{6}$ We provide a formula which expresses the equilibrium flows in terms of centralities of the links.

For the extraction game, a complete network of commons, where all users are connected to all sources, is equivalent to the case of a single "big" common source (Proposition 1). The complete networks add no complexity. At incomplete networks, the missing links differentiate the outcome of a multiple source problem from that of a single source. An absent link harms both the city which misses it and all the cities she shares sources with. The shared sources are over-exploited, like in a standard tragedy of commons. In a network, those sources which are shared by many, sparsely-connected cities suffer more from over-exploitation, where as those which are shared by few, well-connected cities fare better.

We next characterize the efficient amounts of extractions (Proposition 5). Again, the efficient levels depend on the network. Generically, there exists a continuum of efficient flows, which all give the same amounts of total extractions by cities and outflows from sources. To calculate these efficient amounts, we decompose the network into regions (Proposition 5). Each region is a subgraph of the original network. They are cut out from the network according to the ratio of sources to cities in them.

We do not explicitly deal with the question of management of the commons. But the network decomposition we provide is such that in each of the subnetworks we obtain, the problem of efficiency is equivalent to the case of one source and many users (Propositions 3 and 4). Hence with the help of our decomposition, any solution which is proposed for the tragedy of commons ${ }^{7}$ can be applied to a network of commons.

We bridge two branches of the literature. On one side we study a tragedy of commons. We extend the basic model of a common to a network of users and sources. The symmetry between the users is lost (except for exceptional networks like the complete network, the hub, etc.). Given a network, we show how users' equilibrium extraction levels and the efficient distribution of resources is determined by the network structure.

Although we use the metaphor of water, this paper differs from the literature on sharing a river (Ambec and Sprumont 2001; Ni and Wang 2007). The sources in our model work quite differently from a flowing river. Moreover, we do not make any cooperative analysis of the problem.

The other related line of literature is the analysis of behavior on networks. Ballester et al. (2006) analyzes the equilibrium activities at each node of an undirected and simple network. They show that the equilibrium levels are given by a network centrality index, which is similar to the Katz-Bonacich centrality. Ballester and Calvó-Armengol (2009) shows that the first order equilibrium conditions of games which exhibit cross influences between agents' actions are linear complementarity problems. In those two papers, they show that it is the centralities of the nodes which determine the equilibrium outcomes. Our model is the first where the centralities of their links determine the behavior of the nodes.

\footnotetext{
6 As the network is a bi-partite graph, all cycles have even length. Hence any two given links have always either an odd or an even number of nodes between them, independent of the path.

7 See Moulin (1990), Shin and Suh (1997), Ellis and van den Nouweland (2006) for efficient mechanisms. Seabright (1993) gives a survey of the literature on the management of the commons.
} 
The model we introduce can also be used to analyze Cournot competition among firms which are linked through markets. If we think of cities as firms with quadratic costs, and sources as markets with linear demands, the results in this paper show what the equilibrium quantities would be in such a setup. The efficiency in our story would be equivalent to the profit maximization of a cartel that the suppliers might form in a network.

The basic notation, some of which we borrow from Corominas-Bosch (2004), is introduced in Sect. 2. We define and study the extraction game in Sect. 3 and characterize the efficient outcomes in Sect. 4. Section 5 discusses the results. The proofs are given in the Appendix.

\section{Notation}

There are $n$ sources $s_{1}, \ldots, s_{n}$, and $m$ cities $c_{1}, \ldots, c_{m}$. They are embedded in a network that links cities with sources, and cities can acquire their water from the sources they are connected to. We will represent the network as a graph.

A non-directed bipartite graph $g=\langle S \cup C, L\rangle$ consists of a set of nodes formed by sources $S=\left\{s_{1}, \ldots, s_{n}\right\}$, and cities $C=\left\{c_{1}, \ldots, c_{m}\right\}$ and a set of links $L$, each link joining a source with a city. A link from $s_{i}$ to $c_{j}$ will be denoted as $(i, j)$. We say that a source $s_{i}$ is linked to a city $c_{j}$ if there is a link joining the two. We will use $(i, j) \in g$ meaning that $s_{i}$ and $c_{j}$ are connected in $g$. Let $r(g)$ be the number of links in $g$.

A graph $g$ is connected if there exists a path linking any two nodes of the graph. Formally, a path linking nodes $s_{i}$ and $c_{j}$ will be a collection of $t$ cities and $t$ sources, $t \geq 0, s_{1}, \ldots s_{t}, c_{1}, \ldots, c_{t}$ among $S \cup C$ (possibly some of them repeated) such that

$$
(i, 1),(1,1),(1,2), \ldots,(t, t),(t, j) \in g
$$

A subgraph $g_{0}=\left\langle S_{0} \cup C_{0}, L_{0}\right\rangle$ of $g$ is a graph such that $S_{0} \subseteq S, C_{0} \subseteq C, L_{0} \subseteq L$ and such that each link in $L$ that connects a source in $S_{0}$ with a city in $C_{0}$ is a member of $L_{0}$. Hence a node of $g_{0}$ will continue to have the same links it had with the other nodes in $g_{0}$. We will write $g_{0} \subseteq g$ to mean that $g_{0}$ is a subgraph of $g$. For a subgraph $g_{0}$ of $g$, we will denote by $g-g_{0}$, the subgraph of $g$ that results when we remove the set of nodes $S_{0} \cup C_{0}$ from $g$.

Given a subgraph $g_{0}=\left\langle S_{0} \cup C_{0}, L_{0}\right\rangle$ of $g$, let $\overleftrightarrow{g_{0}}$ be the complete bipartite graph with nodes $S_{0} \cup C_{0}$. We call $\overleftrightarrow{g_{0}}$ the completed graph of $g_{0}$.

We will denote by $N_{g}\left(s_{i}\right)$ the set of cities linked with $s_{i}$ in $g=\langle S \cup C, L\rangle$, more formally:

$$
N_{g}\left(s_{i}\right)=\left\{c_{j} \in C \text { such that }(i, j) \in g\right\}
$$

and similarly $N_{g}\left(c_{j}\right)$ stands for the set of sources linked with $c_{j}$.

For a set $A$, let $|A|$ denote the number of elements in $A$. 


\section{The extraction game}

Given a graph $g$, each city $c_{j}$ maximizes her utility by extracting a non-negative amount of water through its links from the sources in $N_{g}\left(c_{j}\right)$. So, the set of players are the set of cities $C$.

We denote by $q_{i j} \geq 0$ the amount of water extracted by city $c_{j}$ from source $s_{i}$.

Now we define the column vector that shows the quantities flowing at each link. Given a graph $g$, let $Q_{g}$ be the column vector of quantities extracted. Hence $Q_{g}$ is the link by link profile of extractions and has size $r(g)$.

For the two graphs given below (Fig. 2)

$$
Q_{g_{1}}=\left[\begin{array}{l}
q_{11} \\
q_{21} \\
q_{12} \\
q_{22}
\end{array}\right] \quad Q_{g_{2}}=\left[\begin{array}{l}
q_{11} \\
q_{21} \\
q_{22}
\end{array}\right]
$$

In the vector $Q_{g}$, the flow $q_{i j}$ is listed above the flow $q_{k l}$ when $j<l$ or when $j=l$ and $i<k$. We will make use of graphs $g_{1}$ and $g_{2}$ in many examples throughout the paper.

Let $\mathbb{Q}^{r}$ be the set of all non-negative real valued column vectors of size $r$. Given a vector of flows $Q_{g}$, for a city $c_{j}$, we will denote by $q_{j}$ the total amount extracted by $c_{j}$. For a source $s_{i}$ we will denote by $q_{i}$ the total outflow from $s_{i}$.

The set of strategies of a city $c_{j}$ is $\mathbb{Q}_{j}$. We denote a representative strategy of $c_{j}$ by $Q_{j} \in \mathbb{Q}_{j}$. Given that there are $r(g)$ links in $g$, the strategy space of the game is $\mathbb{Q}_{g}=\prod_{c_{j} \in C} \mathbb{Q}_{j}=\mathbb{Q}^{r(g)}$. We denote a representative strategy profile on a graph $g$ by $Q_{g} \in \mathbb{Q}_{g}$.

The utility of city $c_{j}$ is $u_{j}\left(Q_{g}\right)$. We will assume that the utility functions of players are additively separable into a concave value and a convex cost of extraction. We will use quadratic value and cost functions, which will decrease the computational load and help us focus on the effects of the network structure. For a given $Q_{g} \in \mathbb{Q}^{r}$, for $\alpha, \beta, \gamma>0$,

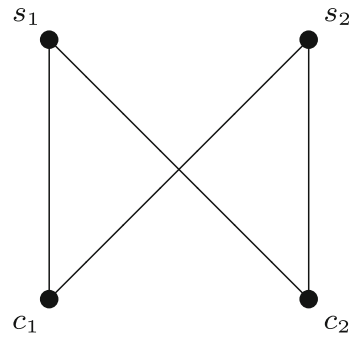

$g_{1}$

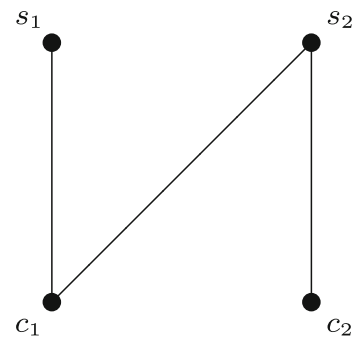

$g_{2}$

Fig. 2 Two different networks of two cities and two sources 


$$
u_{j}\left(Q_{g}\right)=\alpha q_{j}-\frac{\gamma}{2} q_{j}^{2}-\beta \sum_{s_{i} \in N_{g}\left(c_{j}\right)} q_{i j} q_{i}
$$

The first two terms give the value of total extraction.

$$
v_{j}\left(q_{j}\right)=\alpha q_{j}-\frac{\gamma}{2} q_{j}^{2}
$$

The marginal value of extraction is a linear and strictly decreasing function of $q_{j}$. The parameter $\gamma$ is the slope of the marginal value.

The third term in the utility function is the cost of extraction summed over the sources connected to $c_{j}$. We assume that at each $s_{i}$, the cost of extraction is a convex quadratic function

$$
T_{i}\left(Q_{g}\right)=\beta q_{i}^{2}
$$

and assume that each player pays her share of the cost proportional to her extraction. Hence, the cost of extraction $q_{i j}$ by $c_{j}$ from $s_{i}$ is

$$
T_{i j}\left(Q_{g}\right)=\beta q_{i j} q_{i}
$$

The utility function, although separable in terms of the value and the cost of extraction,

$$
u_{j}\left(Q_{g}\right)=v_{j}\left(q_{j}\right)-\sum_{s_{i} \in N_{g}\left(c_{j}\right)} T_{i j}\left(Q_{g}\right)
$$

is not separable with respect to each source. The marginal utility from $q_{i j}$ does depend on the amounts extracted by $c_{j}$ from sources other than $s_{i}$.

The best response $Q_{j}^{\prime}$ of city $c_{j}$ to $Q_{g} \in \mathbb{Q}_{g}$ is such that,

for all links $(i, j), q_{i j}^{\prime}= \begin{cases}\frac{\alpha-\gamma \sum_{s_{l} \in N_{g}\left(c_{j}\right) \backslash\left\{s_{i}\right\}} q_{l j}-\beta \sum_{c_{k} \in N_{g}\left(s_{i}\right) \backslash\left\{c_{j}\right\}} q_{i k}}{2 \beta+\gamma}, & \text { if }\left.\frac{\partial u_{j}}{\partial q_{i j}}\right|_{Q_{g}} \geq 0 \\ 0, & \text { if }\left.\frac{\partial u_{j}}{\partial q_{i j}}\right|_{Q_{g}}<0\end{cases}$

Given a matrix $M \in \mathbb{R}^{t \times t}$ and a vector $p \in \mathbb{R}^{t}$, the linear complementarity problem $L C P(p ; M)$ consists of finding a vector $z \in \mathbb{R}^{t}$ satisfying

$$
\begin{aligned}
z & \geq 0, \\
p+M z & \geq 0, \\
z^{T}(p+M z) & \geq 0
\end{aligned}
$$

Samelson (1958) shows that a linear complementarity problem $L C P(p ; M)$ has a unique solution for all $p \in \mathbb{R}^{t}$ if and only if all the principal minors of $M$ are positive. Given a graph $g$, the first order equilibrium conditions of the extraction game form 
a linear complementarity problem where $z=Q_{g}, p=-\alpha \mathbf{1}_{r(g)}$, where $\mathbf{1}_{r(g)}$ is a vector of 1's of size $r(g)$ and $M$ is the square matrix of the linear system of equations formed by the best response functions of users. ${ }^{8}$ This matrix will be formally defined in the Appendix before the proofs. We prove that it is positive-definite, hence there exists a unique solution.

We further check for the second order conditions for the agents, which reveal that the solution of the linear complementarity problem is indeed the equilibrium of the game.

\section{Theorem 1 The extraction game has a unique Nash equilibrium.}

Example 1 Suppose we have graph $g_{1}$. Let $\alpha=\beta=\gamma=1$. Then the link flows at equilibrium $^{9}$ are $q_{11}^{*}=q_{21}^{*}=q_{12}^{*}=q_{22}^{*}=0.2$.

Suppose the graph was $g_{2}$. Now at equilibrium, $q_{11}^{*}=0.2857, q_{21}^{*}=0.1429$, and $q_{22}^{*}=0.2857$. The deletion of the link $(1,2)$ changes the extraction levels. Now $c_{2}$ is connected only to $s_{2}$ and exploits it more. This makes the extractions from $s_{2}$ more costly and $c_{1}$ extracts less from this source. Instead, $c_{1}$ relies more on her exclusive connection $s_{1}$ where the extraction is less costly.

Let $Q_{g}^{*}$ be an equilibrium of the extraction game. There might be some links in $g$ which carry zero flow at equilibrium $Q_{g}^{*}$. Marginal utilities of extractions from those links need not be zero at $Q_{g}^{*}$.

$$
\begin{aligned}
& q_{i j}^{*}>0 \Rightarrow \frac{\partial u_{j}}{\partial q_{i j}}=0 \\
& q_{i j}^{*}=0 \Rightarrow \frac{\partial u_{j}}{\partial q_{i j}} \leq 0
\end{aligned}
$$

To calculate the equilibrium quantities, first we need to weed out the links with zero flow. We denote by $g-Z\left(Q_{g}^{*}\right)$ the graph obtained from $g$ by deleting the links which have zero flow at $Q_{g}^{*}$.

Theorem 2 Given two graphs $g$ and $g^{\prime}$. Let $Q_{g}^{*}$ and $Q_{g^{\prime}}^{*}$ be the equilibrium of the extraction game in $g$ and $g^{\prime}$, respectively. If $g-Z\left(Q_{g}^{*}\right)=g^{\prime}-Z\left(Q_{g^{\prime}}^{*}\right)$, then $Q_{g-Z\left(Q_{g}^{*}\right)}^{*}=Q_{g^{\prime}-Z\left(Q_{g^{\prime}}^{*}\right.}^{*}$.

At equilibrium there might be links which carry no flows. For the cities of such links, the marginal utilities of extraction through them are not positive. They are indifferent between having such a link or not. Theorem 2 tells us such links play no role in determining the equilibrium. They are strategically redundant. Take graph $g_{3}$ (Fig. 3). Let $\alpha=\beta=\gamma=1$. Then at equilibrium,

Now we cut the link $(1,3)$ and denote the new graph by $g_{3}-(1,3)$ (Fig. 4).

For $\alpha=\beta=\gamma=1$, according to Theorem 2 the flows at equilibrium in both graphs are $q_{11}^{*}=q_{12}^{*}=\frac{1}{4}$ and $q_{23}^{*}=q_{33}^{*}=\frac{1}{4}$. At the equilibrium in $g_{3}$, the marginal

\footnotetext{
8 The matrix is formed by the upper part of the conditional equations as given in (1).

9 The calculations are given in the Appendix.
} 
Fig. 3 A link carries zero flows at equilibrium

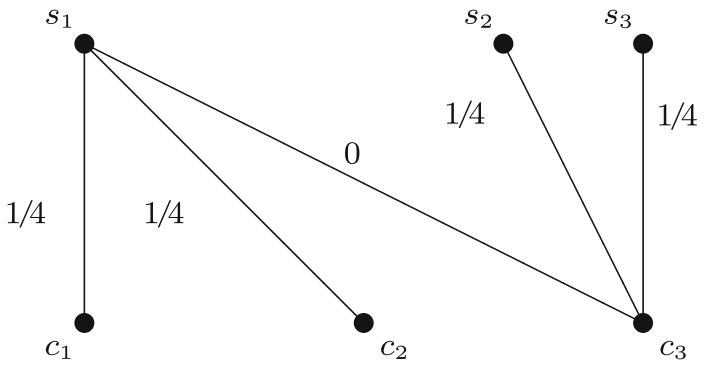

$g_{3}$

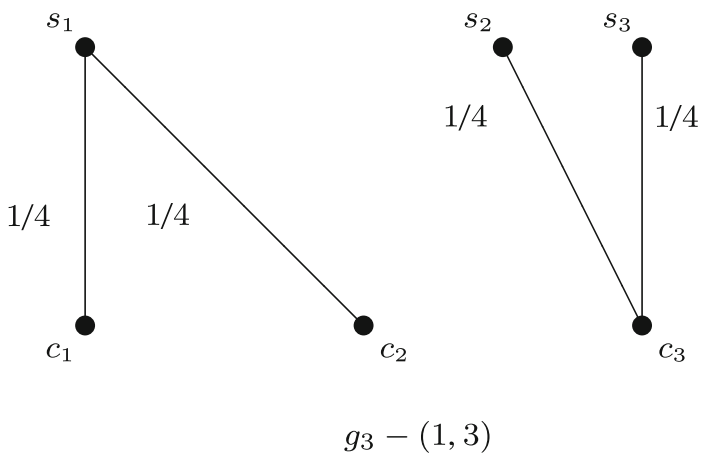

utility to $c_{3}$ from extraction via $(1,3)$ was negative. Deleting it does not change the equilibrium quantities on other links, because the marginal utility on them is the same as in graph $g_{3}$.

We will use the marginal utility argument employed above to give a network interpretation for the flows at equilibrium. Given a graph $g$, we will calculate the equilibrium at graph $g-Z\left(Q_{g}^{*}\right)$. By Theorem 2, this gives us the equilibrium flows in $g$ for the links which carry positive quantities and the equilibrium flows on the rest of the links in $g$ are zero.

Definition 1 Given a graph $g$, a line graph $L(g)$ of $g$ is a graph obtained by denoting each link in $g$ with a node in $L(g)$ and connecting two nodes in $L(g)$ if and only if the corresponding links in $g$ meet at one endpoint.

For the graph $g$, the line graph ${ }^{10} L(g)$ represents the adjacencies between the links in $g$.

For graph $g$ with $k$ nodes, the $k$-square adjacency matrix represents which nodes are connected to which other nodes in $g$. To calculate the equilibrium flows, we will use the adjacency matrix of the line graph $L\left(g-Z\left(Q_{g}^{*}\right)\right)$ of the graph $g-Z\left(Q_{g}^{*}\right)$. This adjacency matrix keeps track of the connections between the links of the network.

\footnotetext{
10 The line graph is also called the edge graph, the adjoint graph, the interchange graph, or the derived graph of $g$.
} 
Given a graph $g$, let $G^{*}$ be the weighted adjacency matrix of the line graph of $g-Z\left(Q_{g}^{*}\right)$ such that the links which are connected via a city have weight $\gamma$ and the links which are connected via a source have weight $\beta$. The columns and the rows in $G^{*}$ correspond to the links in $g-Z\left(Q_{g}^{*}\right)$. If the two links share a city, then the corresponding entry is $\gamma$. If they share a source, then it is $\beta$. For example for graph $g_{2}$ all links have positive flows at equilibrium. Then,

$$
G_{g_{2}}^{*}=\left[\begin{array}{lll}
0 & \gamma & 0 \\
\gamma & 0 & \beta \\
0 & \beta & 0
\end{array}\right]
$$

For any graph $g, G^{*}$ has diagonal entries as 0 and non-diagonal entries are either $0, \gamma$ or $\beta$. We will use $G^{*}$ to denote both the line graph of $g-Z\left(Q_{g}^{*}\right)$ and the weighted adjacency matrix of this graph.

For $a \geq 0$, and a network adjacency matrix $G^{*}$, let

$$
M\left(G^{*}, a\right)=\left[I-a G^{*}\right]^{-1}=\sum_{k=0}^{\infty}\left(a G^{*}\right)^{k}
$$

If $M\left(G^{*}, a\right)$ is non-negative, its entries $m_{i j}\left(G^{*}, a\right)$ counts the number of paths in the network, starting at node $i$ and ending at node $j$, where paths of length $k$ are weighted by $a^{k}$.

Definition 2 For a network adjacency matrix $G$, and for scalar $a>0$ such that $M(G, a)=[I-a G]^{-1}$ is well-defined and non-negative, the vector Katz-Bonacich centralities of parameter $a$ in $G$ is:

$$
\boldsymbol{b}(G, a)=[I-a G]^{-1} . \mathbf{1}
$$

In a graph with $z$ nodes, the Katz-Bonacich centrality of node $i$,

$$
b_{i}(G, a)=\sum_{j=1}^{z} m_{i j}(G, a)
$$

counts the total number of paths in $G$ starting from $i$.

Theorem 3 Given a network of commons $g$, the Nash equilibrium flow vector is

$$
Q_{g-Z\left(Q_{g}^{*}\right)}^{*}=\frac{\alpha}{2 \beta+\gamma}\left[\sum_{k=0}^{\infty}\left(a G^{*}\right)^{2 k} \cdot \mathbf{1}-\sum_{k=0}^{\infty}\left(a G^{*}\right)^{2 k+1} \cdot \mathbf{1}\right]
$$

where $a=\frac{1}{2 \beta+\gamma}$.

The first summation counts the total number of even paths that start from the corresponding node in $G^{*}$, and the second summation counts the total number of odd paths that start from it. 
The first sum tells that the equilibrium extraction from a link is positively related with the number of even length paths that start from it. The flows on links which have an even number of nodes between them are complements. In contrast, the negative sign on the second summation means the equilibrium extraction from a link is negatively related with the number of odd length paths that start from it. The flows on the links which have an odd number of nodes between them are strategic substitutes. This effect of parity in length stems from the fact that all neighboring link flows are substitutes. This makes the flows on links which have a distance of two between them complements. The sign of strategic interaction between the link flows is reversed as the path length between them increases by one.

In the adjacency matrix $G^{*}$ each link has a weight. While counting the number of paths, these weights are taken into account. The extraction by a city $c_{j}$ is calculated by summing up the quantities on the links in $N_{g}\left(c_{j}\right)$. The outflow from a source $s_{i}$ is calculated by summing up the quantities on the links in $N_{g}\left(s_{i}\right)$.

Now we determine a benchmark to demonstrate the effect of the network structure in the game of extractions.

Proposition 1 Let $g$ be a complete bipartite network with $n$ sources and $m$ cities, with the corresponding parameters $\alpha, \beta, \gamma>0$. Let $g^{\prime}$ be the network with a single source and $m$ cities with parameters $\alpha, \frac{\beta}{n}, \gamma>0$. Let $Q_{g}$ and $Q_{g^{\prime}}$ be the equilibrium of the extraction game in $g$ and $g^{\prime}$, respectively. Then the equilibrium consumptions and payoffs in $Q_{g}$ and $Q_{g^{\prime}}$ are the same for all cities in $g$ and $g^{\prime}$.

Proposition 1 establishes the equivalence between a complete bipartite network with multiple sources and a simple commons problem with a single source. The sources in a complete network can be viewed as a single big source. A complete network adds no complexity to the original problem of commons. At incomplete networks the deviations from this benchmark will be due to the structure of the connections.

For example, in graph $g_{3}$, where $\alpha=\beta=\gamma=1$, the equilibrium consumptions and utilities of the cities are

$$
\begin{aligned}
& \left(q_{1}, q_{2}, q_{3}\right)=\left(\frac{1}{4}, \frac{1}{4}, \frac{1}{2}\right) \\
& \left(u_{1}, u_{2}, u_{3}\right)=\left(\frac{3}{32}, \frac{3}{32}, \frac{1}{4}\right) .
\end{aligned}
$$

In the completed graph of $g_{3}$, the equilibrium consumptions and utilities would have been

$$
\begin{aligned}
& \left(q_{1}^{\prime}, q_{2}^{\prime}, q_{3}^{\prime}\right)=\left(\frac{3}{7}, \frac{3}{7}, \frac{3}{7}\right) \\
& \left(u_{1}^{\prime}, u_{2}^{\prime}, u_{3}^{\prime}\right)=\left(\frac{15}{98}, \frac{15}{98}, \frac{15}{98}\right) .
\end{aligned}
$$

The decrease in $c_{1}$ 's and $c_{2}$ 's consumptions and utilities are due to their lack of links in comparison to the complete network and $c_{3}$ benefits as she no longer shares two of the sources with others. 


\section{The efficient extraction}

Assuming that cities have comparable and identical utilities, we will define efficiency as the maximization of the total welfare. But the techniques we introduce can be used to determine the optimal distribution of resources under other possible definitions of efficiency like maximin or weighted utilitarian welfare. ${ }^{11}$

Definition 3 Given a graph $g$, a flow vector $Q_{g}$ is efficient if it maximizes the sum of utilities

$$
U\left(Q_{g}\right)=\sum_{c_{j} \in C} u_{j}\left(Q_{g}\right)=\alpha \sum_{(i, j) \in g} q_{i j}-\frac{\gamma}{2} \sum_{c_{j} \in C}\left(q_{j}\right)^{2}-\beta \sum_{s_{i} \in S}\left(q_{i}\right)^{2}
$$

First, we will characterize the efficient flow vectors in Proposition 2. In a complete bipartite network of commons, due to its symmetry, it is easy to calculate the efficient extractions. We next establish that for a class of networks of commons, the efficient extractions are equal to those in their completed graphs (Propositions 3 and 4). In Proposition 5, we provide a network decomposition to calculate the efficient extractions. Proposition 6 reveals that at equilibrium the sources are exploited more than the efficient level.

Proposition 2 Given a graph $g$, the flow vector $Q_{g}$ is efficient if and only if

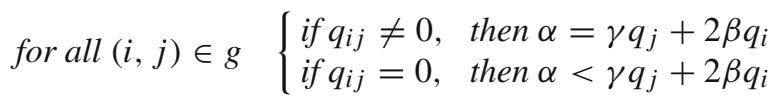

The conditions in Proposition 2 are the first order conditions for efficiency. Since the utility functions are strictly concave in extractions, efficiency is achieved when the resources are distributed as equally as possible within the graph $g$. This means smoothing out of both, extractions among sources, and consumptions among cities. If $Q_{g}^{e}$ is a vector of efficient flows, then for a city $c_{j}$ and any two different sources $s_{i}, s_{k} \in N_{g}\left(c_{j}\right)$

$$
\begin{aligned}
q_{i j}^{e}, q_{k j}^{e} & \neq 0 \Rightarrow q_{i}^{e}=q_{k}^{e} \\
q_{i j}^{e} & =0 \text { and } q_{k j}^{e} \neq 0 \Rightarrow q_{i}^{e}>q_{k}^{e}
\end{aligned}
$$

\footnotetext{
11 See İlkılıç and Kayı (2010) where the techniques we use to calculate efficiency are adopted to determine the outcomes of several allocation rules for a bankruptcy problem on a network.
} 
Similarly, for a source $s_{i}$ and any two different cities $c_{j}, c_{l} \in N_{g}\left(s_{i}\right)$

$$
\begin{aligned}
q_{i j}^{e}, q_{i l}^{e} & \neq 0 \Rightarrow q_{j}^{e}=q_{l}^{e} \\
q_{i j}^{e} & =0 \text { and } q_{i l}^{e} \neq 0 \Rightarrow q_{j}^{e}>q_{l}^{e}
\end{aligned}
$$

We are not guaranteed a unique solution. Indeed, we will see that, in general, there exists a continuum of solutions to the problem of efficient flows. But all efficient flows will lead to the same total extraction for each city and the same total outflow from each source.

Example 2 Suppose we have graph $g_{1}$. Let $\alpha=\beta=\gamma=1$. The the efficient flows are

$$
\begin{aligned}
& \left\{q_{11}^{e}, q_{21}^{e}, q_{12}^{e}, q_{21}^{e} \geq 0: q_{11}^{e}+q_{12}^{e}=\frac{1}{3}, q_{21}^{e}+q_{22}^{e}\right. \\
& \left.=\frac{1}{3}, q_{11}^{e}+q_{21}^{e}=\frac{1}{3} \text { and } q_{12}^{e}+q_{22}^{e}=\frac{1}{3}\right\}
\end{aligned}
$$

There exists a continuum of flows which give an efficient outcome. The total extractions at each city and the total outflows at each source are the same for all the efficient flow levels.

Now we will find a vector of extractions that satisfies the first order conditions of efficiency. Given a subgraph $g_{0}=\left\langle S_{0} \cup C_{0}, L_{0}\right\rangle$ of $g$, consider the efficient amount of extractions and outflows in its completed graph $\overleftrightarrow{g_{0}}$. Clearly the levels are identical across cities and across sources. Let $\overleftarrow{q_{0}}$ be the efficient amount of total extraction by a city in $\overleftrightarrow{g_{0}}$ and $\overrightarrow{q_{0}}$ the efficient amount of total outflow from a source in $\overleftrightarrow{g_{0}}$. If $\left|S_{0}\right|=n_{0}$ and $\left|C_{0}\right|=m_{0}$, then direct calculation shows that

$$
\overleftarrow{q_{0}}=\frac{\alpha n_{0}}{\gamma n_{0}+2 \beta m_{0}} \quad \text { and } \quad \overrightarrow{q_{0}}=\frac{\alpha m_{0}}{\gamma n_{0}+2 \beta m_{0}}
$$

These values depend only on the source/city ratio. For two graphs $g_{0}=\left\langle S_{0} \cup C_{0}, L_{0}\right\rangle$ and $g_{1}=\left\langle S_{1} \cup C_{1}, L_{1}\right\rangle$,

$$
\frac{\left|S_{0}\right|}{\left|C_{0}\right|}=\frac{\left|S_{1}\right|}{\left|C_{1}\right|} \Rightarrow \overleftarrow{q_{0}}=\overleftarrow{q_{1}} \quad \text { and } \quad \overrightarrow{q_{0}}=\overrightarrow{q_{1}}
$$

We will use the efficient levels of the complete graph as benchmarks while calculating the efficient amounts at incomplete bipartite graphs.

Given $g$, we say that a flow vector $Q_{g}$ is feasible if all flows in $Q_{g}$ are non-negative.The set of feasible flow vectors in $g_{0}$ is a subset of the set of feasible flow vectors in its completed graph $\overleftrightarrow{g_{0}}$. Then given efficient levels of extraction $\overleftarrow{q_{0}}$ and outflow $\overrightarrow{q_{0}}$ at $\overleftrightarrow{g_{0}}$, if these amounts are possible in $g_{0}$, then they must be efficient for $g_{0}$ also.

Proposition 3 Let $g_{0}=\left\langle S_{0} \cup C_{0}, L_{0}\right\rangle$ be a subgraph of $g$. If the extraction of $\overleftarrow{q_{0}}$ by each city in $C_{0}$ is possible without exceeding the outflow $\overrightarrow{q_{0}}$ in any source in $S_{0}$, then these levels are efficient in $g_{0}$. 
Fig. 5 Inclusive subgraph

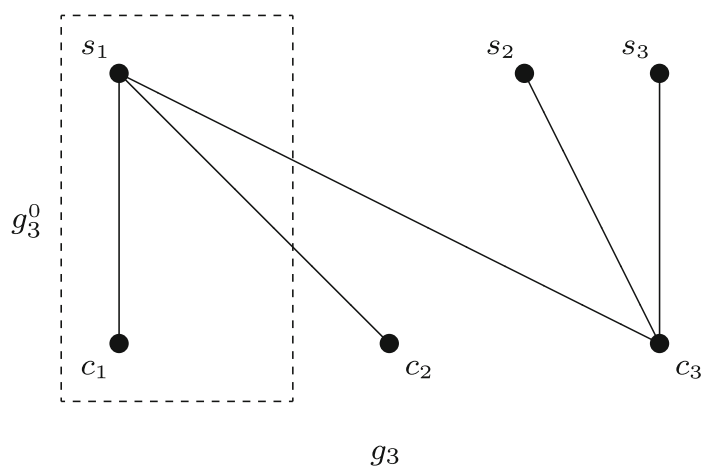

To calculate the efficient flows we need to introduce new graph theoretical definitions.

An inclusive subgraph $g_{0}=\left\langle S_{0} \cup C_{0}, L_{0}\right\rangle$ of $g$ is such that $g_{0}$ is connected and

$$
S_{0}=\bigcup_{c_{j} \in C_{0}} N_{g}\left(c_{j}\right) .
$$

An inclusive subgraph ${ }^{12}$ includes all the sources to which its cities were connected in graph $g$. Let $W(g)=\left\{g_{0} \subseteq g: g_{0}\right.$ is inclusive $\}$ be the set of inclusive subgraphs in $g$. Since $g$ is an inclusive subgraph of itself $W(g) \neq \emptyset$. In graph $g_{3}$ in Fig. 5, the subgraph $g_{3}^{0}$ that we encircle is inclusive. It includes $c_{1}$ and all the sources that $c_{1}$ is connected to.

Given a subset of sources $S_{0} \subseteq S$ and a subset of cities $C_{0} \subseteq C, \frac{\left|S_{0}\right|}{\left|C_{0}\right|}$ is the average number of sources per city. A least inclusive subgraph $\widehat{g}=\langle\widehat{S} \cup \widehat{C}, \widehat{L}\rangle$ of $g$ is such that

$$
\frac{|\widehat{S}|}{|\widehat{C}|}<\frac{|S|}{|C|} \text { and }\langle\widehat{S} \cup \widehat{C}, \widehat{L}\rangle \in \underset{\left\langle S_{0} \cup C_{0}, L_{0}\right\rangle \in W(g)}{\operatorname{argmin}} \frac{\left|S_{0}\right|}{\left|C_{0}\right|}
$$

The first requirement for $\widehat{g}$ to be a least inclusive subgraph of $g$ is for it to have a strictly smaller source/city ratio than $g$. This means that a graph does not necessarily have a least inclusive subgraph. For example a complete bipartite graph has no least inclusive subgraphs. The second requirement is for $\widehat{g}$ to have the smallest source/city ratio among the inclusive subgraphs of $g$. A least inclusive subgraph is inclusive and formed by a set of the least connected cities. There should be no cities in $g$ which are strictly worse than them with respect to source availability.

In Fig. 5, the subgraph $g_{3}^{0}$ is not least inclusive, because the ratio of sources to cities in it is 1 . This ratio for graph $g_{3}$ is also 1 . The subgraph $g_{3}^{1}$ of $g_{3}$, as encircled Fig. 6 , is a least inclusive subgraph. Its source/city ratio is lower than that of $g_{3}$, and there is no other inclusive subgraph of $g_{3}$ with a lower ratio.

\footnotetext{
$\overline{12}$ See Bochet et al. (2009) for the relation between inclusive subgraphs and the Gallai-Edmonds decomposition (Ore 1962) of a bipartite network.
} 
Fig. 6 Least inclusive subgraph

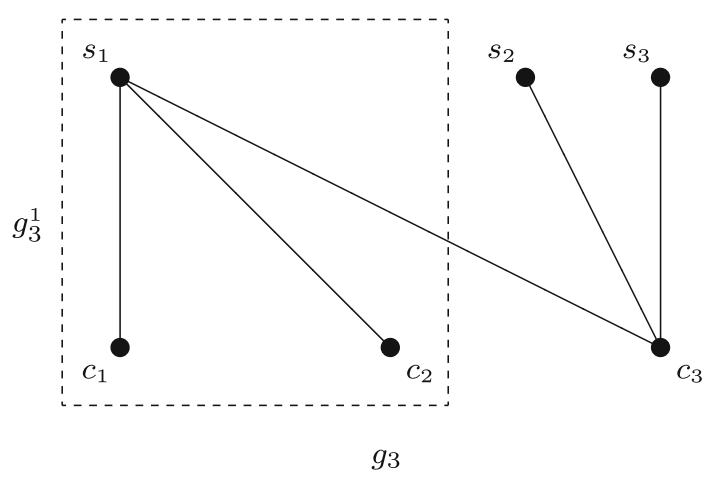

If $\widehat{g}$ is a least inclusive subgraph of $g$, then $\widehat{g}$ cannot have a least inclusive subgraph of its own. Any inclusive subgraph of $\widehat{g}$ is also inclusive in $g$. If $\widehat{g}$ had a least inclusive subgraph with a smaller source/city ratio than $\widehat{g}$, this would have contradicted $\widehat{g}$ having the smallest source/city ratio in $g$.

Now we show that if a subgraph $g_{0}=\left\langle S_{0} \cup C_{0}, L_{0}\right\rangle$ of $g$ has no least inclusive subgraph, then the extraction of $\overleftarrow{q_{0}}$ by each city in $C_{0}$ is possible without exceeding the outflow $\overrightarrow{q_{0}}$ in any source in $S_{0}$

Proposition 4 Let $g_{0}=\left\langle S_{0} \cup C_{0}, L_{0}\right\rangle$ of $g$ be an inclusive subgraph. If $g_{0}$ has no least inclusive subgraph, then the extraction of $\overleftarrow{q_{0}}$ by each city in $C_{0}$ is possible without exceeding the outflow $\overrightarrow{q_{0}}$ in any source in $S_{0}$.

The result means that from an efficiency perspective, if a network of commons has no least inclusive subgraph, it can be treated as a complete network. All the agents are symmetric under efficiency. Hence there is no difference between this problem and the simple tragedy of the commons with a single source. Any solution or mechanism that remedies the latter would solve the tragedy of the commons in a network with no least inclusive subgraph.

We prove Proposition 4 by induction on the number of cities. We start with a city $c_{j}$ of a graph $g_{0}$ with no inclusive subgraphs. This city must be able to extract $\overleftarrow{q_{0}}$, without exceeding the outflow $\overrightarrow{q_{0}}$ in any of its sources. If not, that city with its sources would have formed a least inclusive subgraph in $g_{0}$. Next, we add a new city to this subgraph and iteratively show that such extractions must be possible for all inclusive subgraphs of $g_{0}$ that contain $c_{j}$. As $g_{0}$ is an inclusive subgraph of itself, this proves that such extractions are possible in $g_{0}$.

Decomposing the network. Now we will break down the network of commons $g$, so that the commons problem in each subnetwork is independent from the other ones. We will sequentially cut out least inclusive subgraphs. Hence, they will not have any least inclusive subgraphs of their own. We will continue until we reach a subgraph which has no least inclusive subgraphs. Then in each subgraph, the efficient amounts of total extractions at each city and total outflows at each source will be equal to the efficient amounts in their completed graphs. The next result follows from Propositions 3 and 4. 
Proposition 5 Given a network of commons $g$, the following algorithm calculates the efficient levels of extractions by each city and outflows from each source.

Step 1: Take g. Suppose $g=\langle S \cup C, L\rangle$ has no least inclusive subgraph. Then the efficient total extraction by a city $c_{j}$ and the efficient total outflow from a source $s_{i}$ are equal to the efficient levels in a complete bipartite graph with nodes $S \cup C$, and we are done.

Suppose $g=\langle S \cup C, L\rangle$ has a least inclusive subgraph. Let $g_{0}=\left\langle S_{0} \cup C_{0}, L_{0}\right\rangle$ be the largest least inclusive subgraph ${ }^{13}$ in $\mathrm{g}$. Then, the efficient total extraction by a city $c_{j} \in C_{0}$ is $\overleftarrow{q_{0}}$, and the efficient total extraction from a source $s_{i} \in S_{0}$ is $\overrightarrow{q_{0}}$.

Step 2: Now, for the rest of the cities and sources apply Step 1 to $g-g_{0}$.

In this way we obtain a series of regions out of $g$, with a strictly increasing source per city ratio. In each of them, the efficient levels of extractions would equal to the levels in their respective completed graphs.

So, given a subgraph $g_{0}=\left\langle S_{0} \cup C_{0}, L_{0}\right\rangle$ obtained from the above decomposition, the efficient extraction by a city in $g_{0}$ is

$$
\overleftarrow{q_{0}}=\frac{\alpha n_{0}}{\gamma n_{0}+2 \beta m_{0}}
$$

and the efficient outflow from each source in $g_{0}$ is

$$
\overrightarrow{q_{0}}=\frac{\alpha m_{0}}{\gamma n_{0}+2 \beta m_{0}}
$$

These levels satisfy the first order conditions within each region. Moreover, less resourceful regions have lower amounts of extractions by cities and higher amounts of outflows from sources. Since there are no flows between different regions the first order conditions hold for graph $g$ as well.

The link redundancies reappear while calculating efficiency. Take two graphs $g$ and $g^{\prime}$ such that their decomposition yields the same regions. The efficient amounts of total extractions at each city and total outflows at each source are the same for both $g$ and $g^{\prime}$.

\section{Efficiency versus equilibrium}

If each source were used by a single user, then clearly the Nash equilibrium would be efficient. But when sources are shared, they would be exploited above the efficient levels.

Proposition 6 If there are shared sources, then at equilibrium users extract more than the efficient extraction levels.

Example 3 Suppose we have graph $g_{3}$. Let $\alpha=\beta=\gamma=1$. The decomposition would give us two regions, $g_{3}^{1}$ and $g_{3}-g_{3}^{1}$. Then the efficient flows are

\footnotetext{
13 The ratio $\frac{\left|N_{g}\left(C_{0}\right)\right|}{\left|C_{0}\right|}$ is a submodular function of $C_{0}$, where $N_{g}\left(C_{0}\right)$ is the set of sources connected to $C_{0}$. Then at any graph $g$, there exists a unique largest least inclusive subgraph.
} 


$$
\left\{q_{11}^{e}, q_{12}^{e}, q_{13}^{e}, q_{23}^{e}, q_{33}^{e} \geq 0: q_{11}^{e}=\frac{1}{5}, q_{12}^{e}=\frac{1}{5}, q_{13}^{e}=0, q_{23}^{e}=\frac{1}{4} \text { and } q_{33}^{e}=\frac{1}{4}\right\}
$$

Suppose the graph was $g_{3}-(1,3)$. The decomposition leads to the same regions. The efficient flows are

$$
\left\{q_{11}^{e}, q_{12}^{e}, q_{23}^{e}, q_{33}^{e} \geq 0: q_{11}^{e}=\frac{1}{5}, q_{12}^{e}=\frac{1}{5}, q_{23}^{e}=\frac{1}{4} \text { and } q_{33}^{e}=\frac{1}{4}\right\}
$$

The link $(1,3)$ is redundant from an efficiency point of view, just as it was for equilibrium. The levels of extractions are below the equilibrium for $s_{1}$, which is shared by $c_{1}$ and $c_{2}$ and equal to the equilibrium for $s_{2}$ and $s_{3}$, which are used only by $c_{3}$.

\section{Discussion}

We have analyzed a situation where the tragedy of commons is embedded in a network. We showed that there is a unique equilibrium. This result would hold under less restrictive assumptions on value and cost functions. It would have required the first order conditions to be written as a general(not necessarily linear) complementarity problem. Kolstad and Mathiesen (1987) provide necessary and sufficient conditions for a unique solution to this problem. These conditions can be translated to our setting as "stronger" convexity requirements on the utility functions of cities. ${ }^{14}$

The quadratic cost and value functions, although restrictive, help us focus on the effects of the network on equilibrium. They allow us to formulate the equilibrium quantities in terms of a well-known network centrality index. The quantity flowing through a link is determined by even length and odd length paths starting from it. This effect of parity in length would continue to hold for more general payoff functions, as long as all neighboring link flows are strategic substitutes.

Our analysis paves way for further research. Since the equilibrium is unique, it is possible to study an endogenous network formation game for commons, based on comparative statics. The users in regions with scarce resources would have an incentive to connect to less exploited sources in the network. Another interesting issue is the dynamic exploitation in a network of commons where extractions affect the future availability of the resource. In the static model users depend less on the "crowded" sources. In a dynamic model users might run on such sources before their depletion, aggravating inefficiency.

\section{Appendix}

We first need to introduce additional notation for the proofs.

\footnotetext{
14 An example is Nava (2008) which studies quantity competition in a network of Walrasian agents. With the help of Kolstad and Mathiesen (1987), he provides sufficient conditions on the Jacobians of agents' utility functions for the uniqueness of equilibrium.
} 
Labeling of pairs $(i, j)$

We will order all possible links such that the links of a city $c_{j}$ are assigned a lower number than any city $c_{i}$ for $i>j$, and the links of a city are ordered according to the indices of the sources they come from. The label of a possible link $(i, j)$ will be denoted by $\tau(i, j)$. For example for 2 cities and 2 sources, we will order the links starting from the first city and the first source, $\tau(1,1)=1$. The second link is between the first city and the second source, $\tau(2,1)=2$. Now, as all links of city $c_{1}$ are ranked, $\tau$ will next rank the link between $c_{2}$ and $s_{1}, \tau(1,2)=3$. Then comes the link between city $c_{2}$ and source $s_{2}, \tau(2,2)=4$ (Fig. 7).

For a network $g$, let $Y(g)=\{1 \leq y \leq(n \times m): y=\tau(i, j)$ for some $(i, j) \notin g\}$ be the set of indices that $\tau$ assigns to links which are not in $g$. For 2 cities and 2 sources, for a graph $g$, if the only missing link is $(1,2)$, then $Y(g)=\{3\}$ and $r(g)=3$.

$\tau$ orders all possible links, independent of $g$, where as $Y(g)$ does depend on $g$. We can see how this works on an example. Suppose that 2 cities and 2 sources, form a completely connected bipartite graph $g_{1}$. For graph $g_{1}, Y\left(g_{1}\right)=\emptyset$.

If we cut the link between $c_{2}$ and $s_{1}$, to obtain $g_{2}$, although link $(1,2)$ does not exist in $g_{2}$ it is still labeled equally by $\tau$. $\tau(1,2)=3$, meaning that $Y\left(g_{2}\right)=\{3\}$.

Let $\mathbb{N}_{+}$be the set of positive integers. Let $\rho: L \rightarrow \mathbb{N}_{+}$be a lexicographic order on $L$ respecting $\tau$ such that $\rho$ relabels the $(i, j)$ pairs from 1 to $r(g)$ by skipping those links which are not in $g$.

Explicitly, $\rho: L \rightarrow \mathbb{N}_{+}$is such that:

(i) $\exists(i, j) \in g$ such that $\rho(i, j)=1$,

(ii) $(i, j) \neq(k, l) \Rightarrow \rho(i, j) \neq \rho(k, l)$,

(iii) $j<l \Rightarrow \rho(i, j)<\rho(k, l)$ for all $(i, j),(k, l) \in g$,

(iv) $i<k \Rightarrow \rho(i, j)<\rho(k, j)$ for all $(i, j),(k, j) \in g$,

(v) if $\exists(i, j)$ s.t. $\rho(i, j)=z>1$ then $\exists(k, l) \in g$ s.t. $\rho(k, l)=y-1$.

Let $Z\left(Q_{g}\right)=\left\{1 \leq z \leq r(g): z=\rho(i, j)\right.$ for some $(i, j)$ s.t. $\left.q_{i j}=0\right\}$. Let $\left|Z\left(Q_{g}\right)\right|=t$, then $Q_{g-Z\left(Q_{g}\right)}$ is a vector of size $r(g)-t$ obtained from $Q_{g}$ by deleting the zero entries. It is the vector of quantities for links over which there is a strictly positive flow.

Let $Q_{g}^{*}$ be the equilibrium of the extraction game at network $g$. We denote by $g-Z\left(Q_{g}^{*}\right)$ the network obtained from $g$ by deleting the links which have zero flow

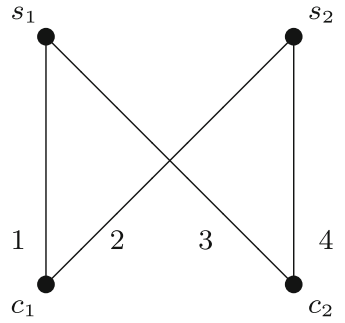

$g_{1}$

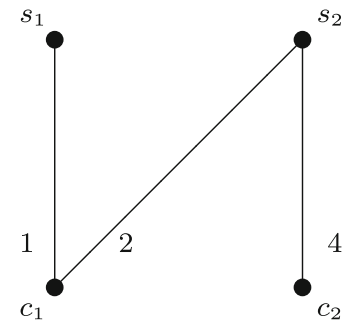

$g_{2}$

Fig. 7 Labeling the links 
at $Q_{g}^{*}$. Let $r^{*}(g)=r(g)-t$. Let $G^{*}=\left[p_{i j}\right]_{r^{*}(g) \times r^{*}(g)}$ be the weighted adjacency matrix of the line graph of $g-Z\left(Q_{g}^{*}\right)$ such that

$$
p_{i j}= \begin{cases}\gamma, & \text { if } \rho^{-1}(i) \text { and } \rho^{-1}(j) \text { share a city } \\ \beta, & \text { if } \rho^{-1}(i) \text { and } \rho^{-1}(j) \text { share a source } \\ 0, & \text { otherwise }\end{cases}
$$

Some useful matrices

Now we define some matrices which we will use in the proofs. We will first introduce auxiliary matrices $A, B, \bar{B}$. These will help us construct the matrices $D$ and $F$. We will use matrices $D$ and $F$ to write the first order conditions for equilibrium and efficiency, respectively, as linear complementarity problems.

For $\beta, \gamma \geq 0$, let $A=\left[a_{i j}\right]_{n \times n}$ be such that,

$$
a_{i j}= \begin{cases}2 \beta+\gamma, & \text { for } i=j \\ \gamma, & \text { for } i \neq j\end{cases}
$$

$A$ has $2 \beta+\gamma$ on the diagonal and $\gamma$ off the diagonal.

$$
A=\left[\begin{array}{cccc}
2 \beta+\gamma & & \\
& \cdot & \gamma & \\
& \cdot & & \\
& \gamma & & 2 \beta+\gamma
\end{array}\right]_{n \times n}
$$

Let $B=\beta I_{n \times n}$, where $I_{n \times n}$ is the identity matrix of size $n$. Using matrices $A$ and $B$, we construct the partitioned matrix $D=\left[d_{i j}\right]_{(m \times n) \times(m \times n)}$ such that:

$$
D=\left[\begin{array}{cccc}
A & & & \\
& \cdot & B & \\
& & \cdot & \\
& B & \cdot & \\
& & & A
\end{array}\right]_{(m \times n) \times(m \times n)}
$$

$D$ has matrix $A$ on its diagonal and matrix $B$ off the diagonal. If we want to write it term by term,

$$
d_{i j}= \begin{cases}2 \beta+\gamma, & \text { for } i=j \\
\gamma, & \text { for } i \neq j, \text { s.t. }(i, j)=\left(z_{1} n+z_{2}, z_{1} n+z_{3}\right) \text { for } z_{1}, z_{2}, z_{3} \in \mathbb{N} \\
\beta, & \begin{array}{l}
\text { s.t. } z_{2} \neq z_{3}, 1 \leq z_{2}, z_{3} \leq n-1 \text { and } z_{1} \leq m-1 \\
\text { for } i \neq j, \text { s.t. } i+j=\left(1+z_{1}\right) n+1+2 z_{2}, \text { for } z_{1}, z_{2} \in \mathbb{N} \\
\text { s.t. } z_{1} \leq m-1, z_{2} \leq m \\
0,
\end{array} \quad \begin{array}{l}
\text { otherwise }
\end{array}\end{cases}
$$


For example for two cities and two sources,

$$
D_{g_{1}}=\left(\begin{array}{cccc}
2 \beta+\gamma & \gamma & \beta & 0 \\
\gamma & 2 \beta+\gamma & 0 & \beta \\
\beta & 0 & 2 \beta+\gamma & \gamma \\
0 & \beta & \gamma & 2 \beta+\gamma
\end{array}\right)
$$

The interpretation, when we use it to find the equilibrium quantities flowing from sources to cities, will be that the column $z$ and the row $z$ in $D$ corresponds to the link $(i, j)$ in $g$ such that $\tau(i, j)=z$. Hence, column 1 and row 1 corresponds to the link $(1,1)$, column 2 and row 2 corresponds to the link $(2,1)$, column 3 and row 3 corresponds to the link $(1,2)$, and column 4 and row 4 corresponds to the link $(2,2)$.

Let $D_{-j}$ be the matrix obtained by deleting row $j$ and column $j$ from $D$. For $J \subset \mathbb{N}_{+}$, let $D_{-J}$ be the matrix obtained by deleting each row $j \in J$ and column $j \in J$ from $D$. We will denote $D_{-Y(g)}$ by $D_{g}$. We obtain $D_{g}$ by deleting each row $y \in Y(g)$ and column $y \in Y(g)$ from $D$. These rows and columns belong to links that are not in $g$. Then, $D_{g}$ has size $r(g) \times r(g)$.

For $g_{2}$, as $Y\left(g_{2}\right)=\{3\}, D_{g_{2}}$ is formed by taking out the third column and third row of $D_{g_{1}}$.

$$
D_{g_{2}}=\left[\begin{array}{ccc}
2 \beta+\gamma & \gamma & 0 \\
\gamma & 2 \beta+\gamma & \beta \\
0 & \beta & 2 \beta+\gamma
\end{array}\right]
$$

Let $\bar{B}=2 B$ be the matrix obtained from $B$ by multiplying it with the scalar 2 . Similarly we construct the partitioned matrix $F=\left[f_{i j}\right]_{(m \times n) \times(m \times n)}$ such that:

$$
F=\left[\begin{array}{cccc}
A & & & \\
& \cdot & \bar{B} & \\
& \cdot & & \\
& \bar{B} & \cdot & \\
& & & A
\end{array}\right]_{(m \times n) \times(m \times n)}
$$

$F$ has matrix $A$ on its diagonal and matrix $\bar{B}$ off the diagonal. If we want to write it term by term,

$$
f_{i j}= \begin{cases}2 \beta+\gamma, & \text { for } i=j \\
\gamma, & \text { for } i \neq j, \text { s.t. }(i, j)=\left(z_{1} n+z_{2}, z_{1} n+z_{3}\right) \text { for } z_{1}, z_{2}, z_{3} \in \mathbb{N} \\
& \begin{array}{l}
\text { s.t. } z_{2} \neq z_{3}, 1 \leq z_{2}, z_{3} \leq n-1 \text { and } z_{1} \leq m-1 \\
\text { for } i \neq j, \text { s.t. } i+j=\left(1+z_{1}\right) n+1+2 z_{2}, \text { for } z_{1}, z_{2} \in \mathbb{N}
\end{array} \\
& \begin{array}{l}
\text { s.t. } z_{1} \leq m-1, z_{2} \leq m \\
\text { otherwise }
\end{array}\end{cases}
$$


For example for two cities and two sources,

$$
F_{g_{1}}=\left[\begin{array}{cccc}
2 \beta+\gamma & \gamma & 2 \beta & 0 \\
\gamma & 2 \beta+\gamma & 0 & 2 \beta \\
2 \beta & 0 & 2 \beta+\gamma & \gamma \\
0 & 2 \beta & \gamma & 2 \beta+\gamma
\end{array}\right]
$$

Similarly, let $F_{-j}$ be the matrix obtained by deleting row $j$ and column $j$ from $F$. For $J \subset \mathbb{N}_{+}$, let $F_{-J}$ be the matrix obtained by deleting each row $j \in J$ and column $j \in J$ from $F$. We will denote $F_{-Y(g)}$ by $F_{g}$. We obtain $F_{g}$ by deleting each row $y \in Y(g)$ and column $y \in Y(g)$ from $F$. These rows and columns belong to links that are not in $g$. Then, $F_{g}$ has size $r(g) \times r(g)$.

For $g_{2}$, as $Y\left(g_{2}\right)=\{3\}, F_{g_{2}}$ is formed by taking out the third column and third row of $F_{g_{1}}$.

$$
F_{g_{1}}=\left[\begin{array}{ccc}
2 \beta+\gamma & \gamma & 0 \\
\gamma & 2 \beta+\gamma & 2 \beta \\
0 & 2 \beta & 2 \beta+\gamma
\end{array}\right]
$$

We show that for $\beta, \gamma>0, D_{g}$ is positive definite, and $F_{g}$ is positive semi-definite for any network $g$. These results (Propositions 7 and 8) will be used in the proofs of Theorem 1 and Proposition 2, respectively.

Proposition 7 For $\beta, \gamma>0, D_{g}$ is positive definite for any network $g$.

Proof of Proposition 7 Let $g$ has $n$ sources, $m$ cities and $r(g)$ links. We show that for the matrix $D_{g}$ we can find a matrix $R_{g}$ with independent columns such that $D_{g}=$ $R_{g}^{T} R_{g}{ }^{15}$ We will write columns of $R_{g}$ so that the entries in $D_{g}$ appear in square roots in $R_{g}$. For example, let us take $D_{g_{3}}$.

$$
D_{g_{3}}=\left[\begin{array}{ccc}
2 \beta+\gamma & \gamma & 0 \\
\gamma & 2 \beta+\gamma & \beta \\
0 & \beta & 2 \beta+\gamma
\end{array}\right]
$$

We write $R_{g_{3}}$ as

$$
R_{g_{3}}=\left[\begin{array}{ccc}
\sqrt{\beta} & 0 & 0 \\
0 & \sqrt{\beta} & 0 \\
0 & 0 & \sqrt{\beta} \\
\sqrt{\gamma} & \sqrt{\gamma} & 0 \\
0 & 0 & \sqrt{\gamma} \\
0 & \sqrt{\beta} & \sqrt{\beta} \\
\sqrt{\beta} & 0 & 0
\end{array}\right]
$$

15 This is equivalent to checking that $D_{g}$ is positive definite. For other characterizations of positive definiteness see Strang (1988). 
Then clearly $D_{g_{3}}=\left(R_{g_{3}}\right)^{T} R_{g_{3}}$. Now, we generalize this to all possible $D_{g}$.

For $s_{i}$ in $S$, we denote $\left|N_{g}\left(s_{i}\right)\right|$ by $m_{i}(g)$. Similarly for $c_{j} \in C$, let $\left|N_{g}\left(c_{j}\right)\right|=$ $n_{j}(g)$, be the number of sources connected to $c_{j}$.

The matrix $D_{g}$ has size $r(g) \times r(g)$. Now, let $R_{g}=\left[r_{i j}\right]_{3 r(g) \times r(g)}$ be such that,

$$
r_{i j}= \begin{cases}\sqrt{\beta}, & \text { for } i=j \\ \sqrt{\frac{\gamma}{n_{z_{1}}(g)},}, & \text { for } i \neq j, \\ & \text { s.t. }(i, j)=\left(\sum_{0 \leq k<z_{1}} n_{k}(g)+r(g)+z_{2}, \sum_{0 \leq k<z_{1}} n_{k}(g)+z_{3}\right) \\ & \text { for } z_{1}, z_{2}, z_{3} \in \mathbb{N} \text { s.t. } 1 \leq z_{2}, z_{3} \leq n_{z_{1}}(g) \text { and } 1 \leq z_{1} \leq m \\ \sqrt{\frac{\beta}{m_{z_{1}}(g)},} & \text { for } i \neq j, \\ & \text { s.t. }(i, j)=\left(\sum_{0 \leq k<z_{1}} m_{k}(g)+z_{2}+2 r(g), \sum_{k=0}^{k=z_{3}} m_{z_{3}}(g)+1\right), \\ & \text { for } z_{1}, z_{2}, z_{3} \in \mathbb{N} \text { s.t. } 1 \leq z_{1}, z_{3} \leq m, \text { and } 1 \leq z_{2} \leq m_{z_{1}}(g) \\ & \text { otherwise }\end{cases}
$$

Let $K_{g}=\beta I_{r(g) \times r(g)}$, for $i \in\{1, \ldots, m\}$ and $L_{i}=\sqrt{\frac{\gamma}{n_{i}(g)}} \mathbf{1}_{n_{i}(g)}$, where $\mathbf{1}$ is the square matrix of 1's. And for $k \in\{1, \ldots, m\}$, we define $\left[m_{i j}^{k}\right]_{(r(g)) \times n_{k}(g)}$ such that,

$$
m_{i j}^{k}= \begin{cases}\sqrt{\frac{\beta}{m_{z_{1}}(g)}}, \quad & \text { for } i \neq j, \\ & \text { s.t. }(i, j)=\left(\sum_{0 \leq k<z_{1}} m_{k}(g)+z_{2}, z_{1}+1\right), \text { for } z_{1}, z_{2} \in \mathbb{N} \\ & \text { s.t. } 1 \leq z_{1} \leq m, \text { and } 1 \leq z_{2} \leq m_{z_{1}}(g) \\ & \text { otherwise }\end{cases}
$$

Then $R_{g}$ can be written as a partitioned matrix,

$$
R_{g}=\left[\begin{array}{cccc} 
& K_{g} & \\
L_{1} & & & \\
& \cdot & 0 & \\
& \cdot & \\
& 0 & \cdot & \\
& & & L_{m} \\
M^{1} & \ldots & M^{k}
\end{array}\right]_{(3 r(g)) \times r(g)}
$$

As $K_{g}$ is a diagonal matrix of size $r(g)$, the row space of $R_{g}$ has dimension $r(g)$, meaning that the column space also has dimension $r(g)$. Then the columns of $R_{g}$ are linearly independent. It is straight forward to check that $D_{g}=\left(R_{g}\right)^{T} R_{g}$.

Proposition 8 For $\beta, \gamma>0, F_{g}$ is positive semi-definite for any network $g$. 
Proof of Proposition 8 The matrix $F_{g}$ has size $r(g) \times r(g)$. Now, let $R_{g}=$ $\left[r_{i j}\right]_{[3(r(g))] \times(r(g))}$ be such that,

$$
r_{i j}= \begin{cases}\sqrt{\frac{\gamma}{n_{z_{1}}(g)},}, & \text { for } i \neq j, \\ & \text { s.t. }(i, j)=\left(\sum_{0 \leq k<z_{1}} n_{k}(g)+r(g)+z_{2}, \sum_{0 \leq k<z_{1}} n_{k}(g)+z_{3}\right) \\ & \text { for } z_{1}, z_{2}, z_{3} \in \mathbb{N} \text { s.t. } 1 \leq z_{2}, z_{3} \leq n_{z_{1}}(g) \text { and } 1 \leq z_{1} \leq m \\ \sqrt{\frac{2 \beta}{m_{z_{1}}(g)},}, & \text { for } i \neq j, \\ & \text { s.t. }(i, j)=\left(\sum_{0 \leq k<z_{1}} m_{k}(g)+z_{2}+2 r(g), \sum_{k=0}^{k=z_{3}} m_{z_{3}}(g)+1\right), \\ & \text { for } z_{1}, z_{2}, z_{3} \in \mathbb{N} \text { s.t. } 1 \leq z_{1}, z_{3} \leq m, \text { and } 1 \leq z_{2} \leq m_{z_{1}}(g) \\ & \text { otherwise }\end{cases}
$$

Let $L_{i}=\sqrt{\frac{\gamma}{n_{i}(g)}} \mathbf{1}_{n_{i}(g)}$, where $\mathbf{1}$ is the square matrix of 1's. For $k \in\{1, \ldots, m\}$, we define $\left[m_{i j}^{k}\right]_{r(g) \times n_{k}(g)}$ such that,

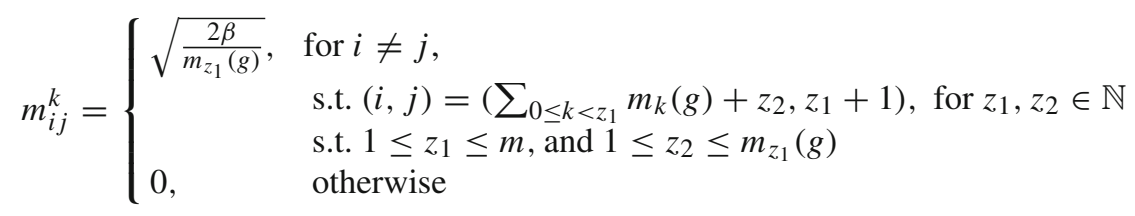

Then $R_{g}$ can be written as a partitioned matrix.

$$
R_{g}=\left[\begin{array}{cccc} 
& & 0 & \\
L_{1} & & & \\
& \cdot & 0 & \\
& \cdot & \\
& 0 & \cdot & \\
& & & L_{m} \\
M^{1} & \ldots & M^{k}
\end{array}\right]_{[3(r(g))] \times(r(g))}
$$

It is straightforward to check that $F_{g}=\left(R_{g}\right)^{T} R_{g}$.

Proof of Theorem 1 Given a graph $g$, the equilibrium conditions of the game is a $L C P\left(-\alpha \mathbf{1}_{r} ; D_{g}\right)$, where $\mathbf{1}$ is a column vector of 1's of size $r$.

$$
\begin{aligned}
Q_{g} & \geq 0, \\
-\alpha \mathbf{1}_{r}+D_{g} Q_{g} & \geq 0, \\
Q_{g}^{T}\left(q+D_{g} Q_{g}\right) & \geq 0
\end{aligned}
$$


Samelson (1958) shows that a linear complementarity problem $L C P(p ; M)$ has a unique solution for all $p \in \mathbb{R}^{t}$ if and only if all the principal minors of $M$ are positive. Positive definite matrices satisfy this condition and we showed in Proposition 7 that $D_{g}$ is positive definite. Then the first order equilibrium conditions have a unique solution.

Let us check the second order condition. For city $c_{k}$, denote the Hessian matrix of the utility $u_{k}$ by $H_{u_{k}}=\left[h_{i j}\right]_{n_{k}(g) \times n_{k}(g)}$ such that

$$
h_{i j}=\left\{\begin{array}{ll}
-2 \beta-\gamma, & \text { for } i=j \\
-\gamma, & \text { for } i \neq j
\end{array} .\right.
$$

We will show that for any $z \in \mathbb{N}_{+}$, the matrix $H_{z}=\left[h_{i j}\right]_{z \times z}$ such that

$$
h_{i j}= \begin{cases}-2 \beta-\gamma, & \text { for } i=j \\ -\gamma, & \text { for } i \neq j\end{cases}
$$

is negative definite.

Let $H_{z}=-(2 \beta+\gamma) H_{z}^{\prime}$, where $H_{z}^{\prime}=\left[h_{i j}^{\prime}\right]_{z \times z}$ is such that,

$$
h_{i j}^{\prime}=\left\{\begin{array}{ll}
1, & \text { for } i=j \\
\phi, & \text { for } i \neq j
\end{array}, \quad \text { where } \phi=\frac{\gamma}{2 \beta+\gamma}\right.
$$

If we denote the determinant of $H_{z}$ by $\operatorname{Det}\left(H_{z}\right)$, then

$$
\operatorname{Det}\left(H_{z}\right)=(2 \beta+\gamma)(-1)^{n} \operatorname{Det}\left(H_{z}^{\prime}\right) .
$$

Now, we show by induction that for all $z \in \mathbb{N}_{+}, \operatorname{Det}\left(H_{z}^{\prime}\right)>0$.

For $z=1, \operatorname{Det}\left(H_{1}^{\prime}\right)=2 \beta+\alpha>0$. Assume $\operatorname{Det}\left(H_{z-1}^{\prime}\right)>0$.

$$
\operatorname{Det}\left(H_{z}^{\prime}\right)=\operatorname{Det}\left(\begin{array}{cccc}
1 & \phi & \cdots & \phi \\
\phi & 1 & \cdots & \cdot \\
\cdot & \cdot & \cdot & \cdot \\
\cdot & \cdot & \cdot & \cdot \\
\cdot & \cdot & & \cdot \\
\phi & \phi & \cdots & \cdot
\end{array}\right)=\left(1-\frac{\phi^{2}(n-1)}{1+(n-2) \phi}\right) \operatorname{Det}\left(H_{z-1}^{\prime}\right)
$$

Then, $H_{z}$ is negative definite and the extraction game with quadratic values has a unique Nash equilibrium.

Calculations of Example 1 Suppose we have graph $g_{1}$. Then

$$
u_{1}\left(Q_{g_{1}}\right)=\left(q_{11}+q_{21}\right)-\frac{\left(q_{11}+q_{21}\right)^{2}}{2}-q_{11}\left(q_{11}+q_{12}\right)-q_{21}\left(q_{21}+q_{22}\right)
$$


Deriving with respect to $q_{11}$ gives

$$
\frac{\partial u_{1}}{\partial q_{11}}=1-3 q_{11}-q_{21}-q_{12}=0
$$

Due to the symmetry of graph $g_{1}, q_{11}^{*}=q_{21}^{*}=q_{12}^{*}=q_{22}^{*}=0.2$. Suppose we have graph $g_{2}$. Then,

$$
u_{1}\left(Q_{g_{2}}\right)=\left(q_{11}+q_{21}\right)-\frac{\left(q_{11}+q_{21}\right)^{2}}{2}-q_{11}^{2}-q_{21}\left(q_{21}+q_{22}\right)
$$

and

$$
u_{2}\left(Q_{g_{2}}\right)=q_{22}-\frac{q_{22}^{2}}{2}-q_{22}\left(q_{21}+q_{22}\right)
$$

Deriving $u_{1}$ with respect to $q_{11}, q_{21}$ and $u_{2}$ with respect to $q_{22}$ gives

$$
\begin{aligned}
& \frac{\partial u_{1}}{\partial q_{11}}=1-3 q_{11}-q_{21}=0 \\
& \frac{\partial u_{1}}{\partial q_{21}}=1-3 q_{21}-q_{11}-q_{22}=0 \\
& \frac{\partial u_{2}}{\partial q_{22}}=1-3 q_{22}-q_{21}=0
\end{aligned}
$$

Solution of the linear equations gives $q_{11}^{*}=0.2857, q_{21}^{*}=0.1429$, and $q_{22}^{*}=0.2857$.

Proof of Theorem 2 Assume $Q_{g-Z\left(Q_{g}\right)}^{*}, Q_{g-Z\left(Q_{g}^{\prime \prime}\right)}^{*}$ are equilibria of the game at $g$ and $g^{\prime}$, respectively. Let $g-Z\left(Q_{g}^{*}\right)=g^{\prime}-Z\left(Q_{g^{\prime}}^{*}\right)$. Then we can write,

$$
D_{g-Z\left(Q_{g}^{*}\right)} \cdot Q_{g-Z\left(Q_{g}^{*}\right)}^{*}=\alpha .1=D_{g^{\prime}-Z\left(Q_{g^{\prime}}^{*}\right)} \cdot Q_{g^{\prime}-Z\left(Q_{g^{\prime}}^{*}\right)}^{*}=D_{g-Z\left(Q_{g}^{*}\right)} \cdot Q_{g^{\prime}-Z\left(Q_{g^{\prime}}^{*}\right)}^{*}
$$

As we showed in Proposition $7 D_{g-Z\left(Q_{g}^{*}\right)}$ is positive definite, hence invertible.

$$
Q_{g-Z\left(Q_{g}\right)}^{*}=Q_{g-Z\left(Q_{g}^{\prime \prime}\right)}^{*}
$$

Proof of Theorem 3 We will calculate the equilibrium flows for the networks $g-Z\left(Q_{g}^{*}\right)$. By Theorem 2, these are equal to the equilibrium flows in $g$ for the links which carry positive flows. The rest of the links in $g$ carry zero flows in equilibrium.

The first order conditions for the equilibrium gives

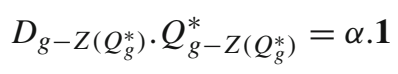


We decompose the matrix $D_{g-Z\left(Q_{g}^{*}\right)}$ using the identity matrix and the matrix $G^{*}$.

$$
\begin{aligned}
D_{g-Z\left(Q_{g}^{*}\right)} \cdot Q_{g-Z\left(Q_{g}^{*}\right)}^{*} & =\left[(2 \beta+\gamma) I+G^{*}\right] \cdot Q_{g-Z\left(Q_{g}^{*}\right)}^{*} \\
& =(2 \beta+\gamma)\left[I+a G^{*}\right] \cdot Q_{g-Z\left(Q_{g}^{*}\right)}^{*}
\end{aligned}
$$

where $a=\frac{1}{2 \beta+\gamma}$. Remember that $Q_{g}^{*}$ is the solution to $L C P\left(-\alpha \mathbf{1}_{r} ; D_{g}\right)$. Then, when we invert $D_{g-Z\left(Q_{g}^{*}\right)}$, the matrix multiplication $\alpha .\left[D_{g-Z\left(Q_{g}^{*}\right)}\right]^{-1} \mathbf{1}$ will give us a strictly positive vector. Now, for $a \geq 0$,

$$
\begin{aligned}
{\left[I+a G^{*}\right] } & =\left[I-a G^{*}\right]^{-1}\left[I-\left(a G^{*}\right)^{2}\right] \\
{\left[I+a G^{*}\right]^{-1} } & =\left[I-\left(a G^{*}\right)^{2}\right]^{-1}\left[I-a G^{*}\right]
\end{aligned}
$$

and

$$
\left[I-\left(a G^{*}\right)^{2}\right]^{-1}=\sum_{k=0}^{\infty}\left(a G^{*}\right)^{2 k}
$$

Substituting this into $D_{g-Z\left(Q_{g}^{*}\right)} \cdot Q_{g-Z\left(Q_{g}^{*}\right)}^{*}=\alpha \cdot \mathbf{1}$,

$$
\begin{aligned}
Q_{g-Z\left(Q_{g}^{*}\right)}^{*} & =a \alpha\left[I-\left(a G^{*}\right)^{2}\right]^{-1}\left[I-a G^{*}\right] . \mathbf{1} \\
& =a \alpha \sum_{k=0}^{\infty}\left(a G^{*}\right)^{2 k}\left[I-a G^{*}\right] . \mathbf{1} \\
& =a \alpha\left[\sum_{k=0}^{\infty}\left(a G^{*}\right)^{2 k} \cdot \mathbf{1}-\sum_{k=0}^{\infty}\left(a G^{*}\right)^{2 k+1} . \mathbf{1}\right]
\end{aligned}
$$

Proof of Proposition 1 In $g$, the utility of $c_{j}$ is

$$
u_{j}\left(Q_{g}\right)=\alpha \sum_{s_{i} \in S} q_{i j}-\frac{\gamma}{2}\left(\sum_{s_{i} \in S} q_{i j}\right)^{2}-\beta \sum_{s_{i} \in S} q_{i j} q_{i}
$$

Then for any link $(i, j), q_{i j}=\frac{\alpha}{n \gamma+(m+1) \beta}$. For any $c_{j}, q_{j}=n q_{i j}=\frac{\alpha}{\gamma+(m+1) \frac{\beta}{n}}$.

In $g^{\prime}$, the utility of $c_{j}^{\prime}$ is

$$
u_{j}^{\prime}\left(Q_{g^{\prime}}\right)=\alpha q_{1 j}-\frac{\gamma}{2} q_{1 j}^{2}-\frac{\beta}{n} \sum_{c_{k} \in C^{\prime}} q_{1 j} q_{1 k}
$$

Then, $q_{1 j}=\frac{\alpha}{\gamma+(m+1) \frac{\beta}{n}}$. Hence, the total consumption by a city is the same in both $g$ and $g^{\prime}$. 
Moreover,

$$
\frac{\beta}{n} \sum_{c_{k} \in C^{\prime}} q_{1 j} q_{1 k}=\beta \sum_{s_{i} \in S} q_{i j} q_{i}
$$

meaning that the utility of a city in $g$ is equal to that of a city in $g^{\prime}$.

Proof of Proposition 2 The first order condition that an efficient vector of flows $Q_{g}^{e}$ has to satisfy is,

$$
\text { for all }(i, j) \in g \quad \begin{cases}\text { if } q_{i j}^{e} \neq 0, & \text { then } \alpha=\gamma q_{j}^{e}+2 \beta q_{i}^{e} \\ \text { if } q_{i j}^{e}=0, & \text { then } \alpha<\gamma q_{j}^{e}+2 \beta q_{i}^{e}\end{cases}
$$

Observe that this is also a linear complementarity problem with $L C P\left(-\alpha \mathbf{1}_{r} ; F_{g}\right) . F_{g}$ is positive semi-definite. Hence, $L C P\left(-\alpha \mathbf{1}_{r} ; F_{g}\right)$ has a solution, though not necessarily unique.

The Hessian matrix of $U$ is $H_{U}=-F_{g}$. By Proposition $8, F_{g}$ is positive semi-definite. Hence, $H_{U}$ is negative semi-definite. Meaning that any $Q_{g}$ that satisfies the first order conditions maximizes $U$.

Proof of Proposition 3 We know that the extraction of $\overleftarrow{q_{0}}$ and the outflow $\overrightarrow{q_{0}}$ satisfies the first order conditions in $\overleftrightarrow{g_{0}}$. Since $g_{0}$ and $\overleftrightarrow{g_{0}}$ have the same set of nodes, they also satisfy the conditions in $g_{0}$.

Proof of Proposition 4 By assumption, $g_{0}$ has no least inclusive subgraphs.

Take a city $c_{j}$ in $g_{0}$. Let $c_{j}$ extract a total of $\overleftarrow{q_{0}}$, such that none of the sources supply more than $\overrightarrow{q_{0}} \cdot \overleftarrow{q_{0}}$ and $\overrightarrow{q_{0}}$ are functions of the source/city ratio. If $c_{j}$ is not linked to enough sources to achieve such an extraction, then city $c_{j}$ and the sources $N_{g}\left(c_{j}\right)$ form a least inclusive subgraph in $g_{0}$, which is a contradiction with $g_{0}$ having no least inclusive subgraphs.

Now, we are going to show by induction that $\overleftarrow{q_{0}}$ extraction by a city in $g_{0}$ such that no source supplies more than $\overrightarrow{q_{0}}$ is possible in any inclusive subgraph of $g_{0}$ that contains $c_{j}$. As $g_{0}$ is an inclusive subgraph of itself, this will imply that such levels of extraction is possible in $g_{0}$.

We know that it is possible for the inclusive subgraph with city $c_{j}$ and the sources $N_{g}\left(c_{j}\right)$. Take an inclusive subgraph $g_{k-1}$ of $g_{0}$ that contains $k-1$ cities including $c_{j}$. Suppose that such levels of extractions are possible in $g_{k-1}$. Denote by $Q_{g_{k-1}}$ such a possible amount of flows in $g_{k-1}$.

Now take an inclusive subgraph $g_{k}$ of $g_{0}$ that contains $k$ cities, $k-1$ which were in $g_{k-1}$ and a fixed city $c_{k}$ which was not in $g_{k-1}$.

Assume that in $g_{k}, \frac{\left|\hat{S}_{k}\right|}{\left|\hat{C}_{k}\right|}<\frac{|\hat{S}|}{|\hat{C}|}$. Then $g_{k}$ is a least inclusive subgraph of $g_{0}$, which is a contradiction.

Then, $\frac{\left|\hat{S}_{k}\right|}{\left|\hat{C}_{k}\right|} \geq \frac{|\hat{S}|}{|\hat{C}|}$. Take $Q_{g_{k-1}}$ which delivers $\overleftarrow{q_{0}}$ to all cities in $g_{k-1}$. As $g_{k}$ contains $g_{k-1}$ we can supply the cities in $g_{k-1}$ with $\overleftarrow{q_{0}}$ without exceeding outflow $\overrightarrow{q_{0}}$ in any source. Now let $c_{k}$ extract through its links such that the outflow from each source in $N_{g}\left(c_{k}\right)$ is $\overrightarrow{q_{0}}$. If the total extraction of $c_{k}$ exceeds $\overleftarrow{q_{0}}$, then we are done. 
If not, denote by $Q^{1}$ the flow vector for $g_{k}$ such that flows for the links which were already in $g_{k-1}$ equals to $Q_{g_{k-1}}$, and the flows for the links which were not in $g_{k-1}$ equals to 0 . Now, given that $c_{k} \notin C_{k-1}$, let ${ }^{16} Q^{2}$ be the flow vector for $g_{k}$ such that

$$
\begin{aligned}
& q_{j k}^{2}=\vec{q}_{0}-q_{i}^{1}, \quad \text { for } j \in N_{g}\left(c_{k}\right) \\
& q_{j l}^{2}=q_{j l}^{1}, \quad \text { for } l \neq k
\end{aligned}
$$

Since $\frac{\left|\hat{S}_{k}\right|}{\left|\hat{C}_{k}\right|} \geq \frac{|\hat{S}|}{|\hat{C}|}$, there must be a source $s_{i}$ in $g_{k}$ not connected to $c_{k}$, such that its outflow in $Q^{2}$ is strictly less than $\overrightarrow{q_{0}}$. Let $S_{k}^{-}$be the set of sources in $g_{k}$ which not connected to $c_{k}$ and which have outflows in $Q^{2}$ strictly less than $\overrightarrow{q_{0}}$.

$$
S_{k}^{-}=\left\{s_{i} \in S_{k}: s_{i} \notin N_{g}\left(c_{k}\right) \text { and } q_{i}^{2}<\overrightarrow{q_{0}}\right\}
$$

Suppose that for any source $s_{i} \in S_{k}^{-}$and for all paths

$$
P=\left\{\left(s_{i}, c_{1}\right),\left(c_{1}, s_{1}\right), \ldots,\left(c_{t}, s_{t}\right),\left(s_{t}, c_{k}\right)\right\}
$$

that connects $s_{i}$ with $c_{k}$, there exists $\left(c_{j}, s_{j}\right) \in P$ such that $q_{j j}^{2}=0$. Given such a path $P$, let $s_{P}$ denote the source $s_{l}$ such that $\left(c_{l}, s_{l}\right) \in P, q_{l l}^{2}=0$ and there exists no other source $s_{j}$ in $P$, closer to $c_{k}$ than $s_{l}$ such that $\left(c_{j}, s_{j}\right) \in P$ and $q_{j j}^{2}=0$. Let $\bar{C}_{k}=\left\{c_{j} \in C_{k}\right.$ : there exists a path $P$ from $s_{i}$ to $c_{k}$ for some $s_{i} \in S_{k}^{-}$and in $P, c_{j}$ is between $s_{P}$ and $c_{k}$ \}. Then the inclusive subgraph with cities $\bar{C}_{k} \cup c_{k}$ is least inclusive in $g_{k}$, which is a contradiction.

Then there exists a source $s_{i} \in S_{k}^{-}$such that there exists a path

$$
P=\left\{\left(s_{i}, c_{1}\right),\left(c_{1}, s_{1}\right), \ldots,\left(c_{t}, s_{t}\right),\left(s_{t}, c_{k}\right)\right\}
$$

that connects $s_{i}$ with $c_{k}$ and $\min _{\left(c_{j}, s_{j}\right) \in P} q_{j j}^{2} \neq 0$. Let

$$
d=\min _{\left(c_{j}, s_{j}\right) \in P}\left\{q_{j j}^{2}, q_{i}^{2}\right\}
$$

Now, given such a path $P$, let $Q^{3}$ be the flow vector for $g_{k}$ such that

$$
\begin{aligned}
q_{i 1}^{3} & =q_{i 1}^{2}+d, \\
q_{j j}^{3} & =q_{j j}^{2}-d, \\
q_{j(j+1)}^{3} & =q_{j(j+1)}^{2}+d \\
q_{t k}^{3} & =q_{t k}^{2}+d \\
q_{l l^{\prime}}^{3} & =q_{l l^{\prime}}^{2}, \text { for all other links }\left(l, l^{\prime}\right)
\end{aligned}
$$

\footnotetext{
16 The subscripts will be used as indices. Hence, for source $s_{i}, q_{i}^{1}$ will denote its outflow at the vector $Q^{1}$.
} 
It is possible to make $c_{k}$ extract at least $\overleftarrow{q_{0}}$ by finding such paths from sources in $\hat{S}_{k}^{-}$ to $c_{k}$ and changing the flows as explained above for each path from a source in $\hat{S}_{k}^{-}$to $c_{k}$. If after using all such paths, $c_{k}$ could still not extract $\overleftarrow{q_{0}}$, then we could use the reasoning above to get a contradiction.

Then the desired levels of extractions are possible in $g_{0}$.

Proof of Proposition 6 We will show that in a subgraph which has no least inclusive subgraphs, at the efficient levels of extractions, every user have links with positive marginal utility of extraction and no links with negative marginal utility of extraction. Since the utilities are strictly concave in extractions, this implies the total extraction for the efficient outcome is less than the equilibrium, where all marginal utilities are zero.

Let $g_{0}=\left\langle S_{0} \cup C_{0}, L_{0}\right\rangle$ be a subgraph which has no least inclusive subgraphs. The efficient total extraction for each city and total outflow from each source in $g_{0}$ are respectively

$$
\overleftarrow{q_{0}}=\frac{\alpha n_{0}}{\gamma n_{0}+2 \beta m_{0}} \quad \text { and } \quad \overrightarrow{q_{0}}=\frac{\alpha m_{0}}{\gamma n_{0}+2 \beta m_{0}}
$$

Assume that $n_{0}<m_{0}$. The efficient outflow from each source is equal. Then for any city $c_{j}$ and link $(i, j)$

$$
\left.\frac{\partial u_{j}}{\partial q_{i j}}\right|_{\overleftarrow{q_{0}}} \geq \frac{\alpha \beta\left(m_{0}-n_{0}\right)}{\gamma n_{0}+2 \beta m_{0}}>0
$$

Assume that $m_{0}<n_{0}$. If some sources are shared, then $m_{0}>1$. The subgraph has no least inclusive subgraphs, hence each user has at least $\frac{n_{0}}{m_{0}}$ links. To achieve the efficient levels, a city $c_{j}$ cannot extract more than $\frac{\alpha m_{0}}{\gamma n_{0}+2 \beta m_{0}}$ from a single source. If $c_{j}$ is extracting $\frac{\alpha m_{0}}{\gamma n_{0}+2 \beta m_{0}}$ from a single source $s_{i}$, then

$$
\frac{\partial u_{j}}{\partial q_{i j}}=0
$$

Then necessarily there exists a source $s_{k}$ and a link $(k, j)$ such that $q_{k j}<\frac{\alpha m_{0}}{\gamma n_{0}+2 \beta m_{0}}$.

$$
\frac{\partial u_{j}}{\partial q_{k j}}>\frac{\partial u_{j}}{\partial q_{i j}}=0
$$

Open Access This article is distributed under the terms of the Creative Commons Attribution Noncommercial License which permits any noncommercial use, distribution, and reproduction in any medium, provided the original author(s) and source are credited. 


\section{References}

Ambec, S., Sprumont, Y.: Sharing a river. J Econ Theory 115, 35-77 (2001)

Ballester, C., Calvó-Armengol, A.: Moderate Interactions in Games with Induced Complementarities. Mimeo: Universitat Autónoma de Barcelona (2009)

Ballester, C., Calvó-Armengol, A., Zenou, Y.: Who's who in networks. Wanted: the key player. Econometrica 75, 1403-1418 (2006)

Benhabib, J., Radner, R.: The joint exploitation of a productive asset. Econ Theory 2, 155-190 (1992)

Bochet, O., İlkılıç, R., Moulin, H.: Egalitarianism under earmark constraints. Mimeo: Maastricht University (2009)

Bonacich, P.: Power and centrality: a family of measures. Am J Sociol 92, 1170-1182 (1987)

Corominas-Bosch, M.: Bargaining in a network of buyers and sellers. J Econ Theory 115, 35-77 (2004)

Dockner, E.J., Sorger, G.: Existence and properties of equilibria for a dynamic game on productive assets. J Econ Theory 71, 209-227 (1996)

Dutta, P.K., Sundaram, R.K.: The tragedy of the commons? Econ Theory 3, 413-426 (1993)

European Council: Regulation No 2371/2002 of 20 December 2002 on the conservation and sustainable exploitation of fisheries resources under the Common Fisheries Policy. Official J Eur Commun 358, 59-80 (2002)

Ellis, C.J., van den Nouweland, A.: A mechanism for inducing cooperation in noncooperative environments: theory and applications. J Public Econ Theory 8, 23-47 (2006)

Gaudet, G., Moreaux, M., Salant, S.W.: Private storage of common property. J Environ Econ Manage 43, 280-302 (2002)

Gordon, H.S.: The economic theory of a common property resource: the fishery. J Polit Econ 62, 124-142 (1954)

İlkılıç, R., Kayı, Ç.: Allocation rules for bankruptcy on a network. Mimeo: Maastricht University (2010)

Katz, L.: A new status index derived from sociometric analysis. Psychometrica 18, 39-43 (1953)

Kremer, M., Morcom, C.: Elephants. Am Econ Rev 90, 212-234 (2000)

Kolstad, C., Mathiesen, L.: Necessary and sufficient conditions for uniqueness of Cournot equilibrium. Rev Econ Studies 54, 681-690 (1987)

Levhari, D., Mirman, L.J.: The great fish war: an example using a dynmic Cournot-Nash solution. Bell J Econ 11, 322-334 (1980)

Moulin, H.: Joint ownership of a convex technology: comparison of three solutions. Rev Econ Stud 57, 439-452 (1990)

Nash, E.: Spain's drought: a glimpse of our future. The Independent 24 May, p 34 (2008)

Nava, F.: Quantity Competition in Networked Markets. Mimeo: University of Chicago (2008)

Ni, D., Wang, Y.: Sharing a polluted river. Games Econ Behav 60, 176-186 (2007)

Ore, O.: Theory of Graphs. American Mathematical Society Colloquium Publications, vol. XXXVIII. Providence: American Mathematical Society (1962)

Ostrom, E.: Governing the Commons. New York: Cambridge University Press (1991)

Ostrom, E., Dietz, T., Dolsak, N., Stern, P.C., Stonich, S., Weber, E.U.: The Drama of The Commons. Washington, D.C.: National Academic Press (2002)

Ostrom, E., Gardner, R., Walker, J.: Rules, Games and Common Pool Resources. Michigan: University of Michigan Press (1994)

Rowat, C., Dutta, J.: The commons with capital markets. Econ Theory 31, 225-254 (2007)

Seabright, P.: Managing local commons: theoretical issues in incentive design. J Econ Perspect 7, 113-134 (1993)

Samelson, H., Thrall, R.M., Wesler, O.: A partition theorem for Euclidean n-space. Proc Am Math Soc 9, 805-807 (1958)

Shin, S., Suh, S.: Double implementation by a simple game form in the commons problem. J Econ Theory 77, 205-213 (1997)

Smith, V.: Economics of production from natural resources. Am Econ Rev 58, 409-431 (1968)

Strang, G.: Linear Algebra and its Applications. San Diego: Harcourt Brace Jovanovich (1988)

Valdecantos, C.: El Congreso deroga el trasvase del Ebro y aprueba el nuevo Plan Hidrológico. El Pais 22 April, p 28 (2005) 